\title{
Étude de l'effet d'un polyacrylate et d'un polyphosphate sur la morphologie et la minéralogie du sulfate de calcium
}

\author{
S. Ben Ahmed ${ }^{1}$, M.M. Tlili ${ }^{1}$, M. Ben Amor ${ }^{1}$ and S. Joiret ${ }^{2}$ \\ 1 Laboratoire de Géochimie et Physico-Chimie de l'Eau, Institut National de Recherche \\ Scientifique et Technique, BP. 95, 2050 Hammam-Lif, Tunisie \\ ${ }^{2}$ Laboratoire Interface et Systèmes Électrochimiques, UPR 15 du CNRS, Université $P$. et \\ M. Curie, 4 place Jussieu, Courrier n¹33, 75252 Paris Cedex 05, France
}

\begin{abstract}
The retarding influence of polyacrylate and polyphosphate on the nucleation of calcium sulphate was investigated. SEM analyses show that these inhibitors didn't only influence the nucleation kinetics but also the precipitate morphology by adsorption onto crystal growth sites. The influence of different concentrations of the additives on the mineralogy of solid precipitated was also examined. Spectroscopy Raman analyses showed that the calcium sulphate can precipitate under different phases, gypsum $\left(\mathrm{CaSO}_{4} \cdot 2 \mathrm{H}_{2} \mathrm{O}\right)$, hemihydrate $\left(\mathrm{CaSO}_{4} \cdot 1 / 2 \mathrm{H}_{2} \mathrm{O}\right)$ and / or anhydride $\left(\mathrm{CaSO}_{4}\right)$, as function of inhibitors concentrations.
\end{abstract}

\section{INTRODUCTION}

L'entartrage par le sulfate de calcium est l'un des problèmes majeurs rencontrés dans le secteur de dessalement des eaux de mer et des eaux saumâtres riche en sulfate [1]. Il existe six phases, anhydres ou hydratées, du sulfate de calcium : le gypse $\left(\mathrm{CaSO}_{4} \cdot 2 \mathrm{H}_{2} \mathrm{O}\right) ; 2$ hémihydrates (Det $\left.\mathrm{E}\right)$ et 3 anhydrites (I, II, III). Toutes ces formes peuvent exister à température ambiante sauf l'anhydrite I qui existe à partir de $1180^{\circ} \mathrm{C}$ [2]. La solubilité de toutes les variétés du sulfate de calcium diminue quand la température augmente. Ceci peut expliquer l'entartrage des échangeurs de chaleurs dans le cas de dessalement thermique [3].

L'inhibition chimique de la précipitation du sulfate de calcium est la méthode la plus utilisée pour remédier à ce problème. Les polyphosphates et les polyacrylates sont les familles de tartrifuges généralement utilisées à cause de leur susceptibilité à former des liaisons avec les cations $\left(\mathrm{Ca}^{2+}\right)$ [4-7]. Ceci leur permet d'être efficace même à faible dose.

L'effet des polyphosphates et des phosphonates sur la précipitation du gypse a été étudié par Liu et Nancollas [4]. Ils ont montré que ces inhibiteurs ralentissent la vitesse de précipitation à partir de solution sursaturée en sulfate de calcium et augmentent le temps de germination. Öner [5] a étudié l'effet de quelques polyélectrolytes. Il a trouvé que les acrylates étaient plus efficace que les méthacrylates. Récemment, El Dahan [6] a testé l'influence d'un ester de phosphate organique. Cet inhibiteur était efficace pour empêcher la précipitation du sulfate de calcium à partir d'une solution synthétique sursaturée. Dans un travail antérieur [7], nous avons testé différents types d'inhibiteurs (polyacrylates, polyphosphates) sur la cinétique de précipitation du sulfate de calcium à 
partir d'une solution sursaturée préparée par la méthode de double décomposition. Nous avons montré que le polyacrylate de sodium est l'inhibiteur le plus efficace. Aussi, nous avons montré que les inhibiteurs testés n'ont pas d'effet sur la nature de la phase précipitée. Le gypse est la phase cristallisée en présence et en absence d'inhibiteurs.

Nous remarquons que dans tous ces travaux, la précipitation a eu lieu à partir de solutions sursaturées. Dans ce travail, nous allons provoquer la précipitation de sulfate de calcium par évaporation d'une solution initialement sous-saturée. Le but est d'étudier l'effet d'un polyacrylate et d'un polyphosphate sur l'étape de la germination, la morphologie et la minéralogie (nature de la phase précipitée) du sulfate de calcium. La caractérisation des phases précipitées est réalisée au moyen du microscope électronique à balayage MEB et par spectroscopie Raman.

\section{PARTIE EXPÉRIMENTALE}

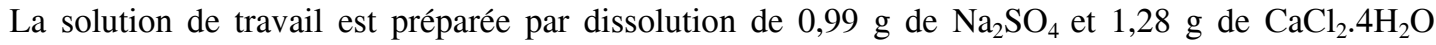
dans un litre d'eau distillée. Le coefficient de sursaturation de cette solution calculé à $50^{\circ} \mathrm{C}$ par la formule (1) est égal à 0,56 . La solution de travail est donc sous-saturée en sulfate de calcium dihydraté.

$$
E \frac{\left(\mathrm{Ca}^{2 \square}\right)\left(\mathrm{SO}_{4}^{2 \square}\right)}{K s}
$$

Où Ks est le produit de solubilité de $\mathrm{CaSO}_{4} \cdot 2 \mathrm{H}_{2} \mathrm{O}$

$(\mathrm{Ca} 2+)$ et (SO42-) sont les activités des ions $\mathrm{Ca} 2+$ et $\mathrm{SO} 42-$ en solution.

Un volume de $400 \mathrm{~mL}$ de cette solution est placé dans un ballon immergé dans un bain thermostaté réglé à la température de travail. Sous pression réduite, on arrive à évaporer la solution même à de faibles températures. La vapeur d'eau est condensée dans un réfrigérant puis récupérée dans une éprouvette graduée. Pour s'assurer de la quantité évaporée, nous déterminons à chaque fois par pesée la masse de la solution restante dans le ballon.

Le sulfate de calcium précipite selon la réaction suivante :

$\mathrm{CaCl}_{2}+\mathrm{Na}_{2} \mathrm{SO}_{4}+2 \mathrm{H}_{2} \mathrm{O} \quad 0 \quad \mathrm{CaSO}_{4} \cdot 2 \mathrm{H}_{2} \mathrm{O}+2 \mathrm{NaCl}$

La germination est détectée suite à la diminution de la concentration en ions calcium libre dans la solution évaporée par rapport à une concentration en ions calcium calculée théoriquement. La concentration des ions $\mathrm{Ca}^{2+}$ est déterminée par dosage complexométrique à l'EDTA.

L'effet d'inhibiteurs chimiques sur l'état thermodynamique de la solution est évalué suite au calcul du coefficient de sursaturation $\mathrm{E}$ (1) au moment de la germination.

Les inhibiteurs testés sont :

- PAS, polyacrylate sodique. L'analyse montre que ce produit contient $68,94 \%$ d'eau et $31,06 \%$ de produit actif.

- Le STPP, TriPolyPhosphate de Sodium de formule chimique $\mathrm{Na}_{5} \mathrm{P}_{3} \mathrm{O}_{10}$. Il présente $100 \%$ de matière active. 
Les solides déposés sont récupérés par filtration et séchés à $40^{\circ} \mathrm{C}$ pour stopper l'hydratation. Ils sont ensuite analysés par spectroscopie Raman et par microscope électronique à balayage MEB.

\section{RÉSULTATS ET DISCUSSION}

\subsection{Effet des inhibiteurs sur la germination}

A une température d'évaporation de $100^{\circ} \mathrm{C}$, les valeurs du coefficient de sursaturation au moment de la précipitation, $\mathrm{E}$, en absence et en présence de $5 \mathrm{mg} . \mathrm{L}^{-1}$ d'inhibiteurs sont présentés dans le tableau suivant :

Tableau 1. Effet des inhibiteurs sur la germination du sulfate de calcium.

\begin{tabular}{|c|c|c|c|}
\hline & Sans inhibiteur & $5 \mathrm{mg} \cdot \mathrm{L}^{-1} \mathrm{STPP}$ & $5 \mathrm{mg} \cdot \mathrm{L}^{-1} \mathrm{PAS}$ \\
\hline $\begin{array}{c}\text { E calculé au point de } \\
\text { germination }\end{array}$ & 1,12 & 1,41 & 1,63 \\
\hline
\end{tabular}

La valeur de $\mathrm{E}$ augmente en présence d'inhibiteurs. On passe de 1,12 à 1,41en présence de STPP et à 1,63 en présence de la même concentration en PAS $\left(5 \mathrm{mg} . \mathrm{L}^{-1}\right)$. Les inhibiteurs retardent la germination et augmente l'étendu du domaine métastable. En effet, ils peuvent intervenir par adsorption sur les premières associations de $\mathrm{Ca}-\mathrm{SO}_{4}$ changeant leur énergie de surface et empêchent ces amas d'atteindre la taille critique des germes. Le polyacrylate, polymère organique, est plus efficace que le polyphosphate minéral. Des effets similaires ont été observés à température plus basse, $50^{\circ} \mathrm{C}$.

\subsection{Effet des inhibiteurs sur la morphologie des cristaux précipités}

Après détection de la précipitation, nous laissons la solution séjourner quelques minutes à la même température pour pouvoir récupérer des cristaux de sulfate de calcium. Ces cristaux sont ensuite analysés par microscope électronique à balayage MEB (figure 1).

Généralement, le gypse cristallise sous forme de bâtonnets : ce sont de longues et fines aiguilles plates et dispersées. La figure 1-a des cristaux précipités en absence d'inhibiteurs illustrent bien cette forme.

A la différence des aiguilles précipitées sans inhibiteurs, la figure 1-b montre qu'en présence de 5 ppm de PAS, le précipité se présente sous forme d'aiguilles accolées par la base. L'adsorption de l'inhibiteur sur les premiers germes engendre une agglomération de ces derniers. Une fois que ces germes atteignent la taille critique, ils poussent sous forme d'aiguilles séparées. L'agrandissement de ces aiguilles (figure 1-c) montre une altération de la morphologie des particules unitaires, des agrégats et des états de surface provenant d'une adsorption des inhibiteurs sur les sites de croissances.

Les inhibiteurs influencent donc non seulement la germination mais aussi la croissance cristalline. 


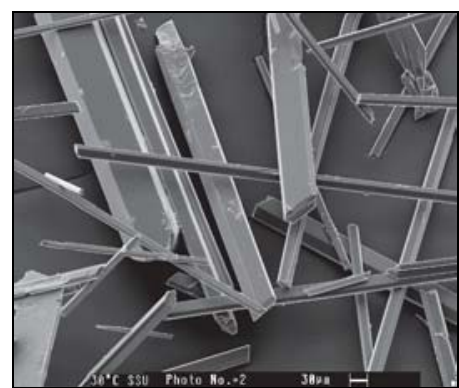

a

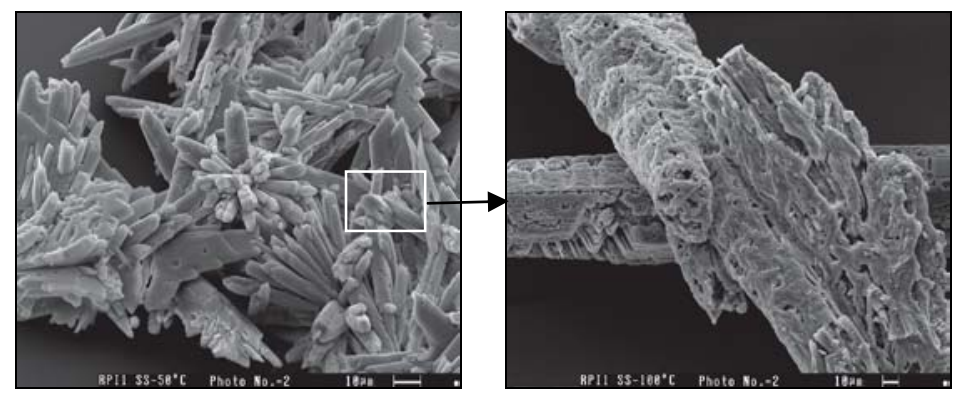

b

$\mathrm{c}$

Figure 1. Photos MEB des cristaux précipités en (a) absence et (b, c) en présence d'inhibiteurs.

\subsection{Effet des inhibiteurs sur la minéralogie des cristaux précipités}

L'analyse Raman des aiguilles présentées sur la figure 1-a montre que le sulfate de calcium précipité est le $\mathrm{CaSO}_{4} \cdot 2 \mathrm{H}_{2} \mathrm{O}$ (figure 2). En effet, le pic principal $\left(1008 \mathrm{~cm}^{-1}\right)$ correspond à celui de vibration de l'ion sulfate dans le réseau du gypse. La présence de l'eau est marquée par les deux pics vers $3400-3500 \mathrm{~cm}^{-1}$.

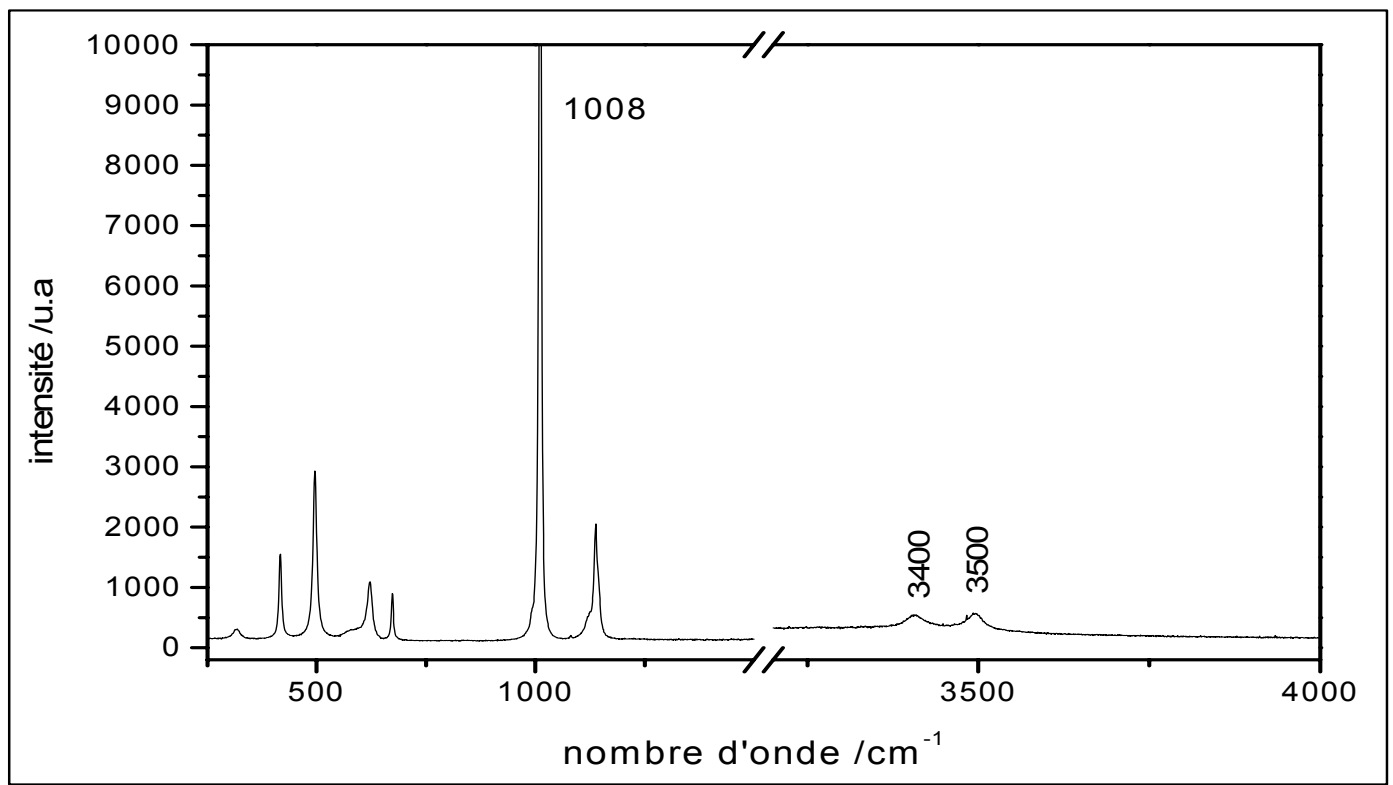

Figure 2. Spectre Raman du précipité obtenu sans inhibiteurs.

Malgré son effet sur la morphologie, les inhibiteurs testés n'influencent pas la phase cristalline si on précipite à une température d'évaporation de $50^{\circ} \mathrm{C}$. Par contre, à $100^{\circ} \mathrm{C}$, l'analyse Raman a permis de distinguer différentes phases en fonction de la quantité d'inhibiteurs ajoutées.

\subsubsection{Effet du polyacrylate}

En présence de $1 \mathrm{mg} . \mathrm{L}^{-1}$ de PAS, l'analyse Raman (figure 3) montre que le sulfate de calcium, précipité à partir de la solution sous-saturée, cristallise sous les trois variétés ; gypse $\left(\mathrm{CaSO}_{4} \cdot 2 \mathrm{H}_{2} \mathrm{O}\right)$, anhydre $\left(\mathrm{CaSO}_{4}\right)$ et hémihydrate $\left(\mathrm{CaSO}_{4} \cdot \frac{1}{2} \mathrm{H}_{2} \mathrm{O}\right)$. En effet, on enregistre un pic intense vers $1017 \mathrm{~cm}^{-1}$ 
caractéristique de la vibration de l'ion sulfate dans le réseau de la forme anhydre. On enregistre aussi deux autres pics, d'intensités moins importantes que ce dernier, vers $1028 \mathrm{~cm}^{-1}$ et $1008 \mathrm{~cm}^{-1}$. Ces deux pics correspondent respectivement aux vibrations de l'ion sulfate dans le réseau de l'hémihydrate et du gypse.

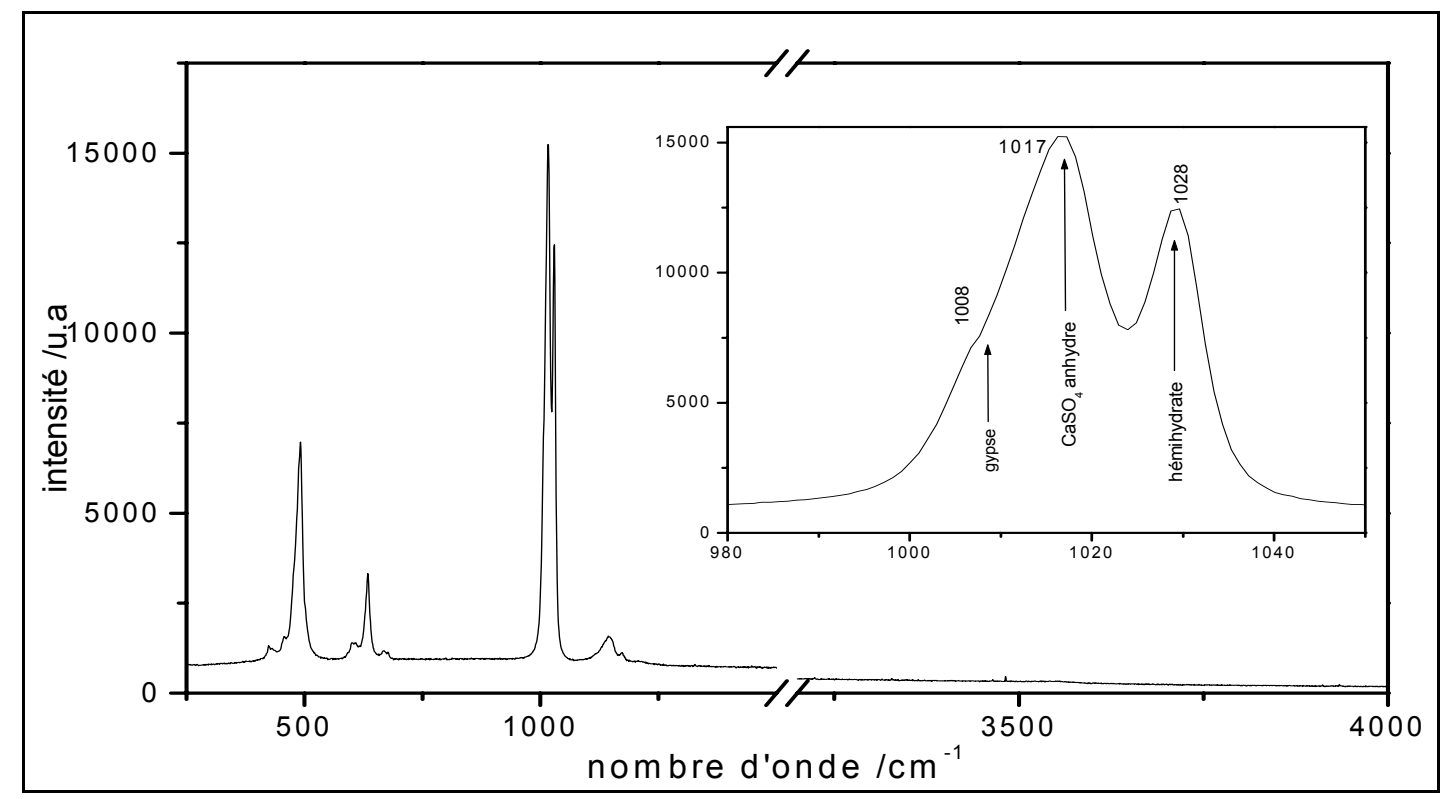

Figure 3. Spectre Raman du précipité obtenu à $100^{\circ} \mathrm{C}$ en présence de $1 \mathrm{mg} . \mathrm{L}^{-1}$ en PAS.

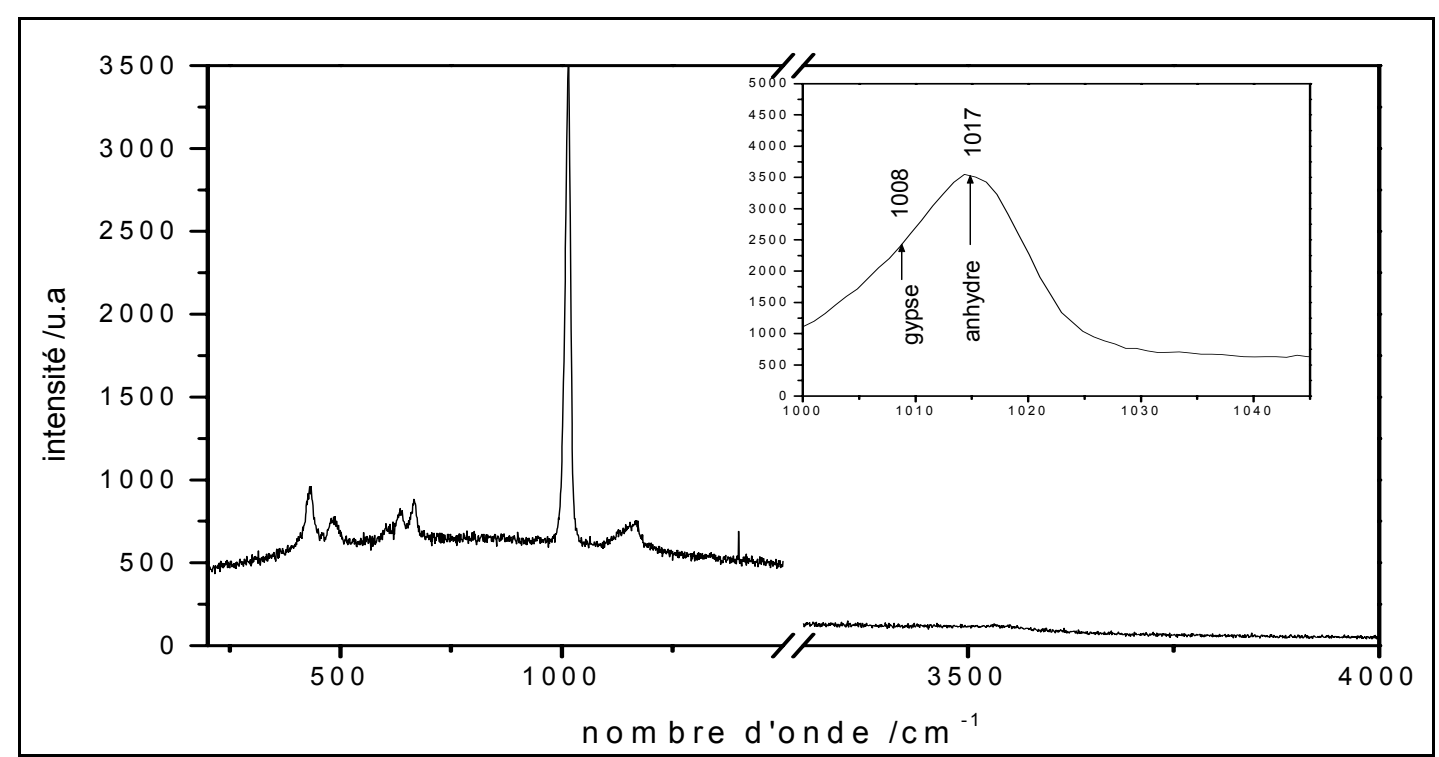

Figure 4. Spectre Raman du précipité obtenu à $100^{\circ} \mathrm{C}$ en présence de 5 mg.L-1 en PAS.

En augmentant la concentration $\left(5 \mathrm{mg} . \mathrm{L}^{-1}\right)$, le polyacrylate (PAS) inhibe la formation de l'hémihydrate qui disparaît et oriente la cristallisation vers la forme anhydre comme le montre le spectre présenté sur 
la figure 4. Le précipité est formé par de l'anhydre et quelque trace de gypse. Sur ce spectre on enregistre une disparition des pics caractéristiques de vibration O-H de l'eau.

\subsubsection{Effet du polyphosphate}

Une concentration de $1 \mathrm{mg} . \mathrm{L}^{-1}$ de STPP n'a pas modifié la structure cristalline du sulfate de calcium précipité. Si on augmente la concentration $\left(5 \mathrm{mg} . \mathrm{L}^{-1}\right)$, on observe des effets similaires à ceux en présence du PAS. En effet, la précipitation du gypse diminue en faveur de la formation de l'anhydre. Le spectre effectué dans ces conditions (figure 5) montre qu'on cristallise les deux variétés : gypse (pic caractéristique vers $1008 \mathrm{~cm}^{-1}$ ) et anhydre (pic caractéristique vers $1017 \mathrm{~cm}^{-1}$ ). La quantité du gypse précipitée dans ces conditions est supérieure à celle précipitée en présence de $5 \mathrm{mg} . \mathrm{L}^{-1}$ de PAS.

Les deux inhibiteurs gênent les molécules de l'eau dans la structure du gypse et empêchent leur intercalation entre les feuillets de $\mathrm{CaSO}_{4}$. Ce qui favorise la précipitation de la forme anhydre aux dépens des formes hydratées.

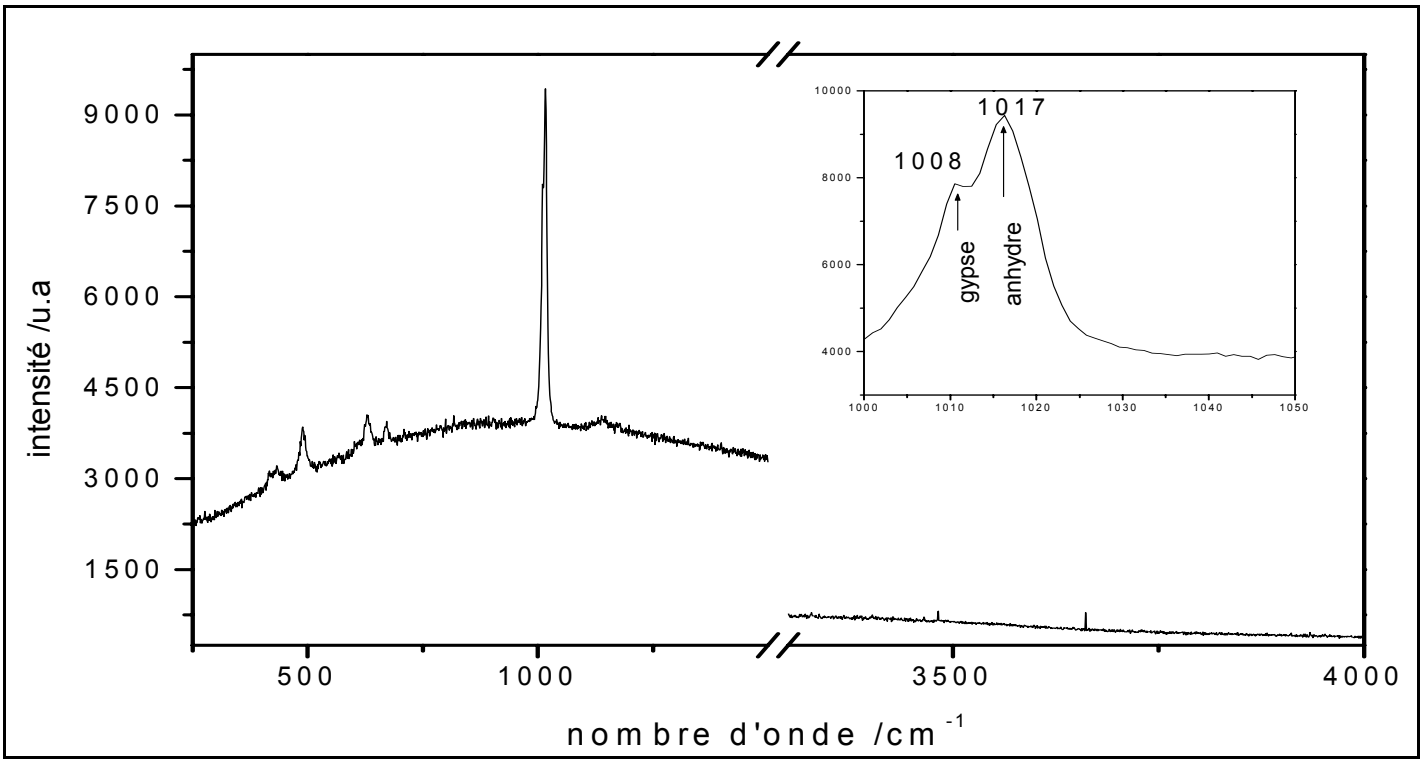

Figure 5. Spectre Raman du précipité obtenu à $100^{\circ} \mathrm{C}$ en présence de $5 \mathrm{mg} . \mathrm{L}-1$ en STPP.

\section{CONCLUSION}

La présence des inhibiteurs chimiques retarde la germination du sulfate de calcium et augmente l'étendu du domaine métastable. Cette inhibition est due à une adsorption sur les premiers clusters qui devraient croître pour donner un germe stable. La croissance est aussi influencée et ce par son adsorption sur certains sites de croissance. La présence de ces inhibiteurs perturbe aussi l'organisation cristalline en influençant le degré d'hydratation.

\section{Remerciements}

Nous remercions Madame Suzane Joiret de l'UPR15 du CNRS à l'Université Pierre et Marie Curie de Paris pour la réalisation et l'interprétation des spectres Raman. 


\section{Références}

[1] A. Maurel, Ph. Audier, H. Barnier, C. Duffau ; "Etude de la solubilité du sulfate de calcium en vue de l'application au dessalement de l'eau de mer et des eaux saumâtres", 1982

[2] T.H. Chong, R. Sheikholeslami; "Thermodynamics and kinetics for mixed calcium carbonate and calcium sulfate precipitation", Chemical Engineering Science 56 (2001) 5391-5400

[3] A. Helalizadeh, H. Muller-Steinhagen, M. Jamialahmadi;"Mixed salt crystallisation fouling", Chemical Engineering and Processing 39 (2000) 29-43

[4] S. T. Liu, G. H. Nancollas, J. Colloid Interface Sci.44 (1973) 422

[5] M. Öner, Ö. Doğan, G. ÖNER; "The influence of polyelectrolytes architecture on calcium sulfate dihydrate growth retardation", J. Crystal growth 186 (1997) 427-437

[6] H.A. El Dahan, H.S. Hegazy ; "Gypsum scale control by phosphate ester", Desalination 127 (1999) 111-118

[7] S. Ben Ahmed, M. Tlili, M. Ben Amor, H. Ben Bacha, B. Elleuch, "Calcium sulphate scale prevention in a desalination unit using the SMCEC technique" Desalination 167 (2004) 311-318 